Math. Model. Nat. Phenom.

Vol. 6, No. 2, 2011, pp. 173-186

DOI: $10.1051 / \mathrm{mmnp} / 20116207$

\title{
The Geometric and Dynamic Essence of Phyllotaxis
}

\author{
P. Atela* \\ Department of Mathematics, Smith College, Northampton, MA 01063, USA
}

\begin{abstract}
We present a dynamic geometric model of phyllotaxis based on two postulates, primordia formation and meristem expansion. We find that Fibonacci, Lucas, bijugate and multijugate are all variations of the same unifying phenomenon and that the difference lies on small changes in the position of initial primordia. We explore the set of all initial positions and color-code its points depending on the phyllotactic type of the pattern that arises.
\end{abstract}

Key words: phyllotaxis, fibonacci, pattern formation, primordia, meristem

AMS subject classification: $00,51,92 \mathrm{C} 80$

\section{Introduction}
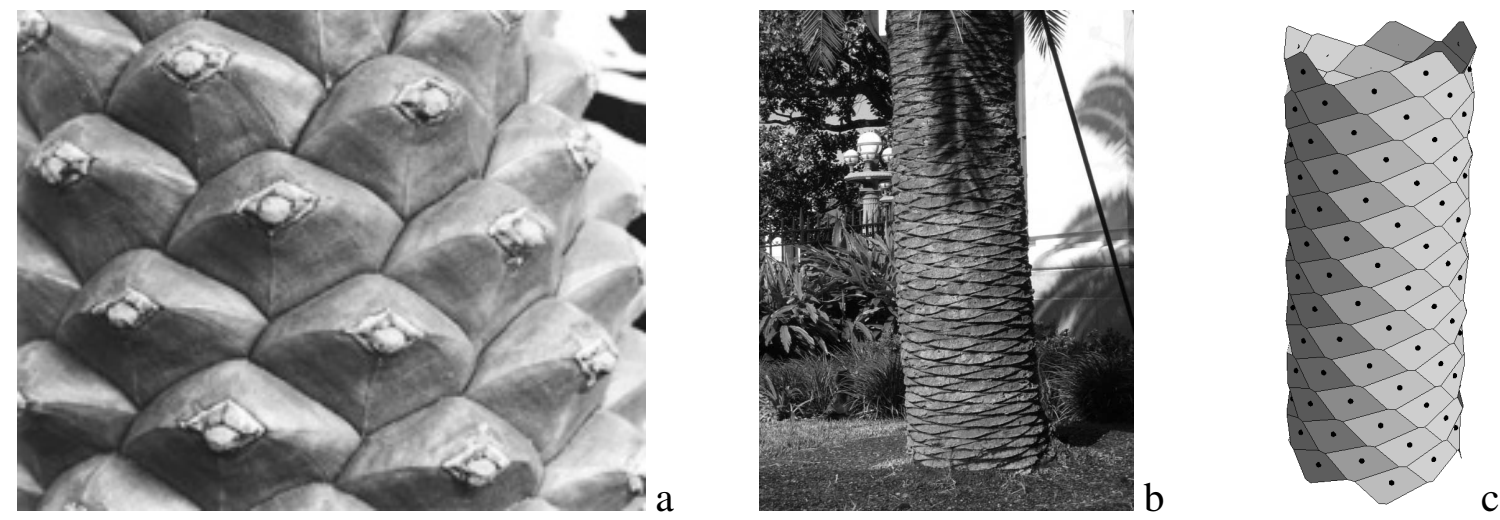

Figure 1: (a) Close view of a pine cone. (b) Palm tree in New Orleans. (c) Mathematical model.

*E-mail: patela@smith.edu 
The aim of this article is to extract the common geometrical and dynamical essence of some of the models of phyllotaxis (pattern formation) that have been studied in the past and propose a simple one, based on two main postulates.

We are basing our model mainly on the studies of Douady and Couder [2, 3], who previously presented a compelling physical experiment involving magnetized drops of ferrofluid into a magnetized dish filled with silicone oil, producing phyllotactic patterns with Fibonacci numbers.

In Botany, a phyllotactic pattern refers to the spiral patterns observed in pine cones, pineapples, asparagus, flowers, palm trees, artichoke, cauliflower, broccoli, lettuce, some cacti, aloe vera, etc. (see Figure 1). These patterns typically come as two visible sets of spirals winding in opposite directions (sometimes a third set is also clearly visible, as in the case of pineapples). These spirals are called parastichies. The surprising phenomenon that interests us here is that when counting the parallel parastichies (spirals) in these two sets, most often one gets two consecutive numbers of the Fibonacci sequence $1,2,3,5,8,13,21,34, \ldots$.. By far, the Fibonacci pairs are the most common in nature. In some few cases two consecutive Lucas numbers $3,4,7,11, \ldots$ also appear. And there are specimens in which one sees consecutive pairs of the sequence $2,4,6,10,16, \ldots$ (socalled Bijugate, two times the Fibonacci numbers), and also Multijugate. In these sequences, each number is the sum of the previous two. (For example, $3+5=8,5+8=13, \ldots$ and also $3+4=7,4+7=11$, etc.) We emphasize that, artificially, one can create a pattern that has any two numbers and it would have an appearance similar to the Fibonacci one in Figure 1c. However, in plants, when there are visible spirals, the pairs of numbers mentioned above are the ones that appear.

Here in this introduction we give a schematic overview of what we understand happens as plants grow, starting at germination, and the geometric effects produced on phyllotactic patterns. We condense the main features into two postulates, which are stated below.
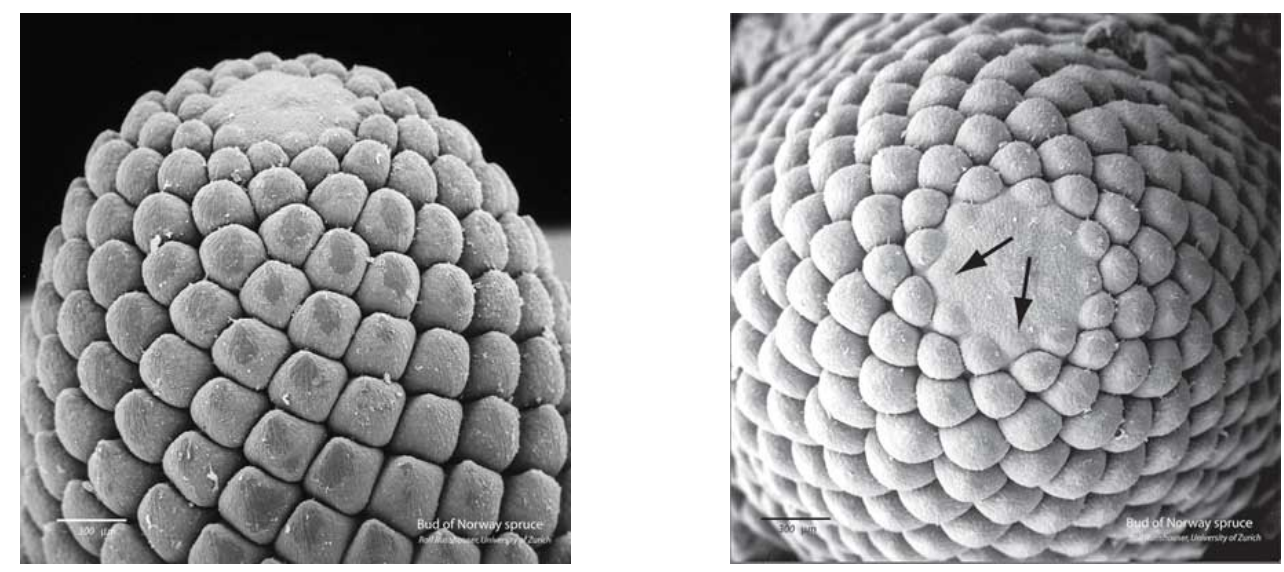

Figure 2: On the left, primordia in a Norway spruce with the meristem at the tip of the shoot. Meristem's diameter is here approx. $500 \mu m$ (Rolf Rutishauser, Univ. of Zurich). On the right, view from the top. The arrows point to two incipient primordia in the active meristem. Notice that they appear "in between" older existing primordia.

As Hofmeister [4] observed, phyllotactic patterns are established at a microscopic scale as the botanical units called primordia appear at the periphery of the apical meristem, piling up on 
the side of the stem as time passes (see Figures 1 and 2). One observes that, after this initial appearance, the whole structure that includes and sustains older primordia expands in size, but the local phyllotactic pattern that has already formed is preserved. In particular, each primordium maintains its neighbors.

Following van Iterson[10], Snow\&Snow[9], Douady\&Couder[3], Atela, Golé \& Hotton[1] and others (see also [6], [7], etc.), we think of the meristem as a circular region at the tip of the stem, which we assume axisymmetric, with primordia forming at its boundary (see Figure 2). It is useful to model primordia as circles with a given radius, but the important thing is the placement of the centers of these circles.

(For implementations of Hofmeister's periodic formation of primordia vs. Snow\&Snow's same size primordia appearing when there is enough space see, for example, Douady\&Couder[3], Atela, Golé \& Hotton[1], also Dumais et al.[5]).

\subsection{Two postulates}

We suggest in this article that it is the dynamic combination of two main biological phenomena that influences phyllotaxis as a plant grows: we call them primordia appearance and meristem expansion. These form the basis of the dynamic geometric model that we present below.

P1. Primordia appearance: Primordia appear with approximately the same size, "in between" older primordia at the periphery of the meristem (see Figures 1 and 2).

In this paper we place primordia systematically by placing them following the principle of "in the first available" spot, the so-called Snow\&Snow model (see [3]). This condition can be relaxed a bit, as the "coin experiment" below shows.

As we mentioned before, after primordia appear and others follow afterwards near them, they start being away from the active meristem and the whole structure that contains and surrounds them expands, as one can see in Figures 1 and 2. So, away from the meristem, older primordia and the diameter of the cross section of the cylindrical shoot continue to expand. However, the phyllotactic pattern near these primordia had already formed and we assume it does not change during this growth, all the way to macroscopic level. (There might be some distortions and/or a lot of tissue growth in between primordia, so that these might seem to grow apart from each other as is the case of, for example, leaves.)

P2. Meristem expansion: We point out a second type of expansion that we think takes place in plants and is crucial for the appearance of Fibonacci numbers (and the few other cases, such as Lucas numbers 3, 4, 7, 11, .., bijugate, etc.). When the seed germinates it starts with few primordia-perhaps one or two - and as new primordia appear, the meristem expands in relation to primordia size. We emphasize that this meristem expansion is a different phenomenon from the expansion mentioned in the previous paragraph which the whole structure further away from the active meristem undergoes. It would be interesting to study in real plants how exactly this expansion occurs and at what stage or stages of meristem development it does. 

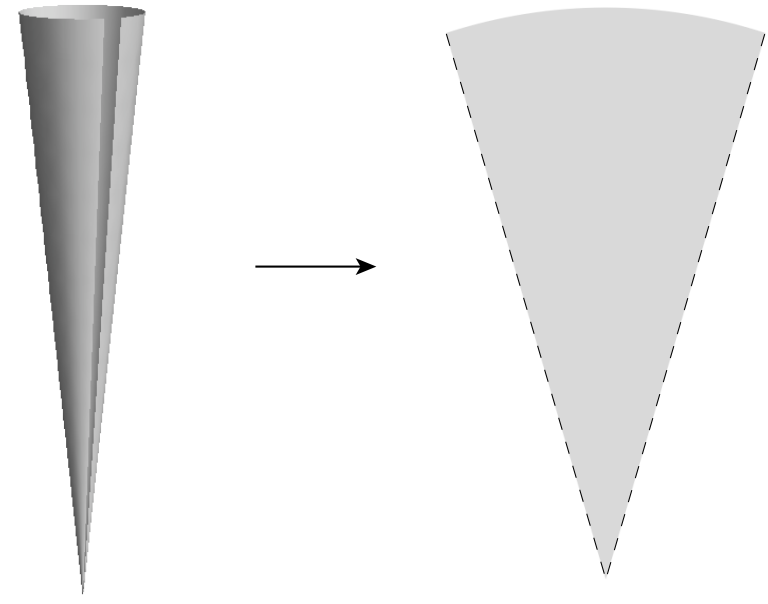

Figure 3: Cutting a cone and unrolling it unto a flat plane gives us a "wedge" shape.

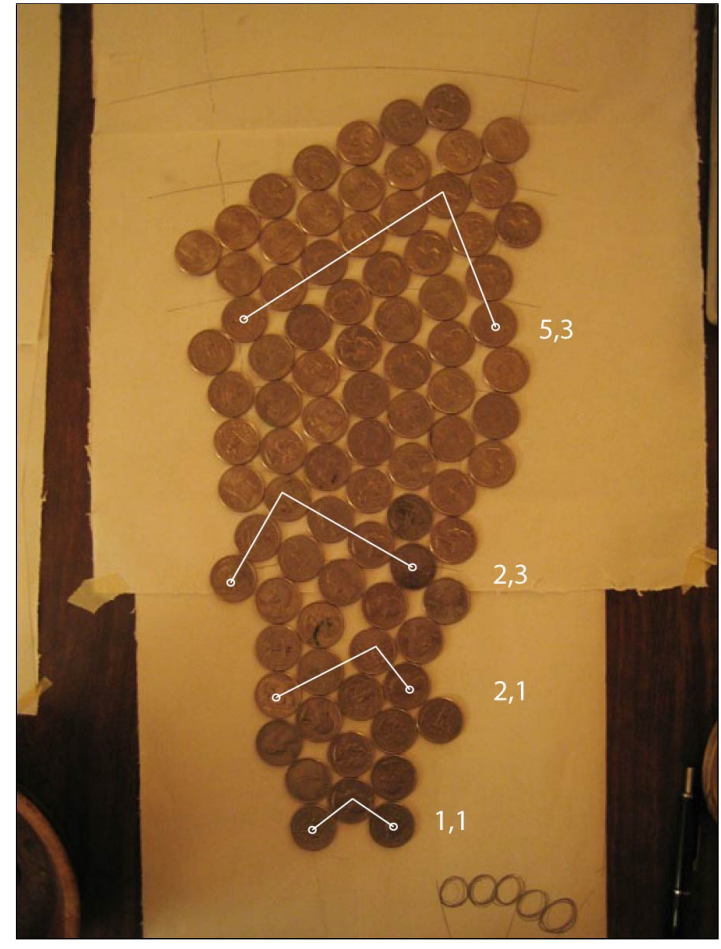

Figure 4: Rising phyllotaxis. Two coins in the lower part represent the same single primordium. Some of the coins on the left boundary have a corresponding one on the right, representing the same primordium.

\section{The Coin Experiment}

We present here a coin experiment modeling plant pattern formation (phyllotaxis) that physically realizes the two postulates, i.e., as the plant grows, primordia appear during meristem expansion, followed by, perhaps, periods of meristem's size stabilization.

Since primordia appear at the outer ring of the circular meristem, we model this expanding ring process with a conic shape, which we will deal with geometrically by cutting it along its side and unrolling it flat on a plane, thus getting a "wedge" as Figure 3 shows.

We carried an experiment with coins as primordia. We roughly drew two straight lines forming a wedge on a piece of paper taped flat on a table, representing an unrolled cone. We then placed two coins on the lower part on each boundary, both representing the same single primordium because the two side boundaries of the wedge should be identified. We then started placing coins, piling them up, by putting new ones "in between" older ones, not too systematically, but usually at the lower spot possible. As we kept placing coins, we observed rising phyllotaxis with Fibonacci numbers appearing as we filled the cone. The photograph taken of the result is in Figure $4 .^{\dagger}$

${ }^{\dagger}$ I carried out this simple experiment in April 2006 and reported it with some of the results of the next sections in the Phyllotaxis session in New Orleans, at the Joint Mathematics Meetings, on Jan 5, 2007. 


\section{The Dynamic Geometric Model}

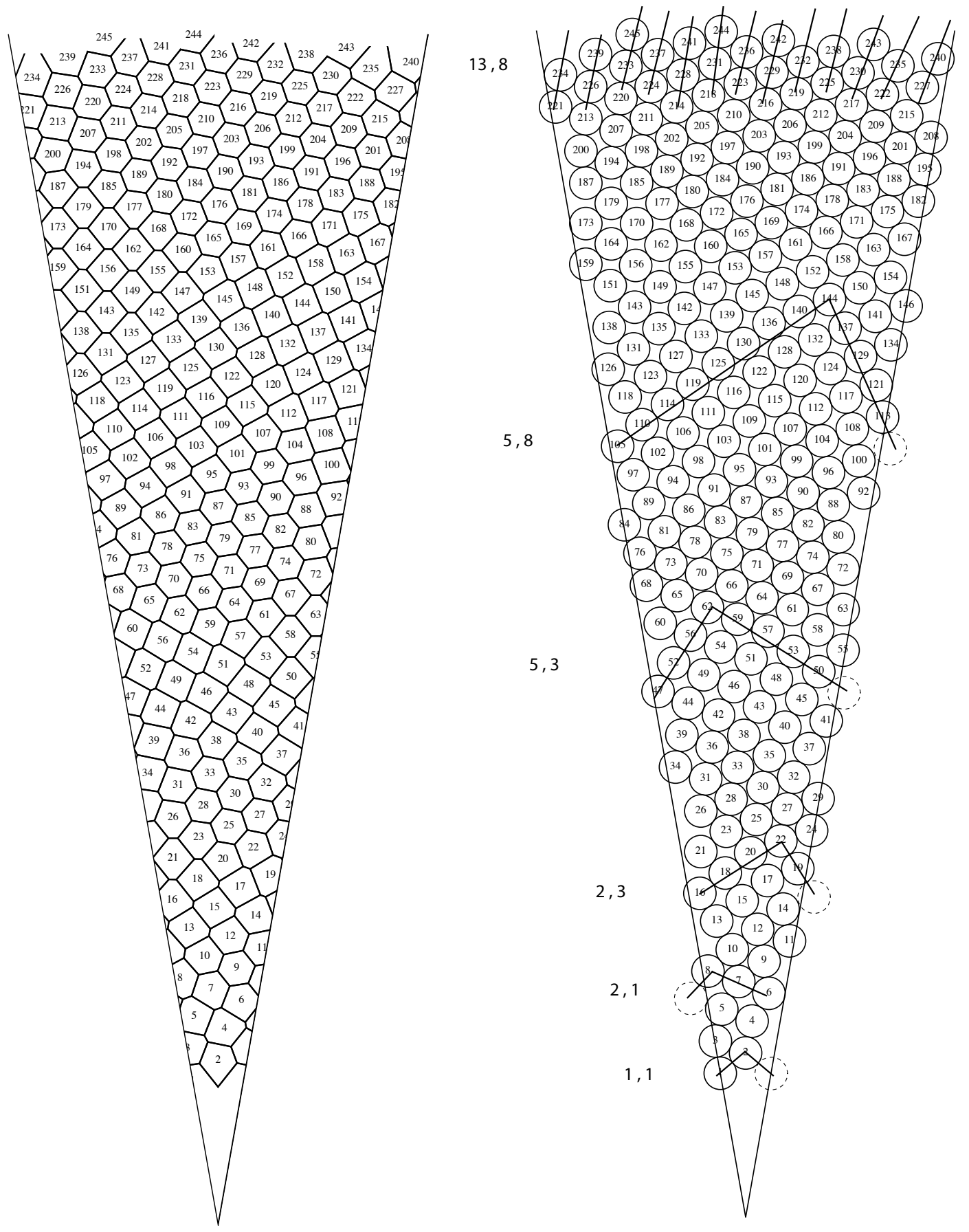

Figure 5: Example with rising Fibonacci phyllotaxis starting with one primordia. The angle here is arbitrarily $20^{\circ}$ (the rest of the paper is always $15^{\circ}$ ). On the left, the voronoi cells.

We report here a systematic numerical study implementing the two postulates of the dynamic geometric model. This model consists of placing circles of the same size on a circular cone, one 
by one, in an iterative process (one could relax a bit the equal size condition). Each new circle placed at the lowest possible spot (towards the vertex) (this condition can also be relaxed) without overlaps with existing ones. Thus, each new circle is tangent to two (sometimes three) previous circles.

Figure 5 shows a reproduction of the coin experiment using a computer. We used here an arbitrary angle of $20^{\circ}$. Everywhere else in this paper we use $15^{\circ}$. The starting circle is placed not too far apart from itself, so that a second one can be placed tangent to it at the lowest spot. Starting with only one circle at other heights in this way resulted always in Fibonacci configurations.
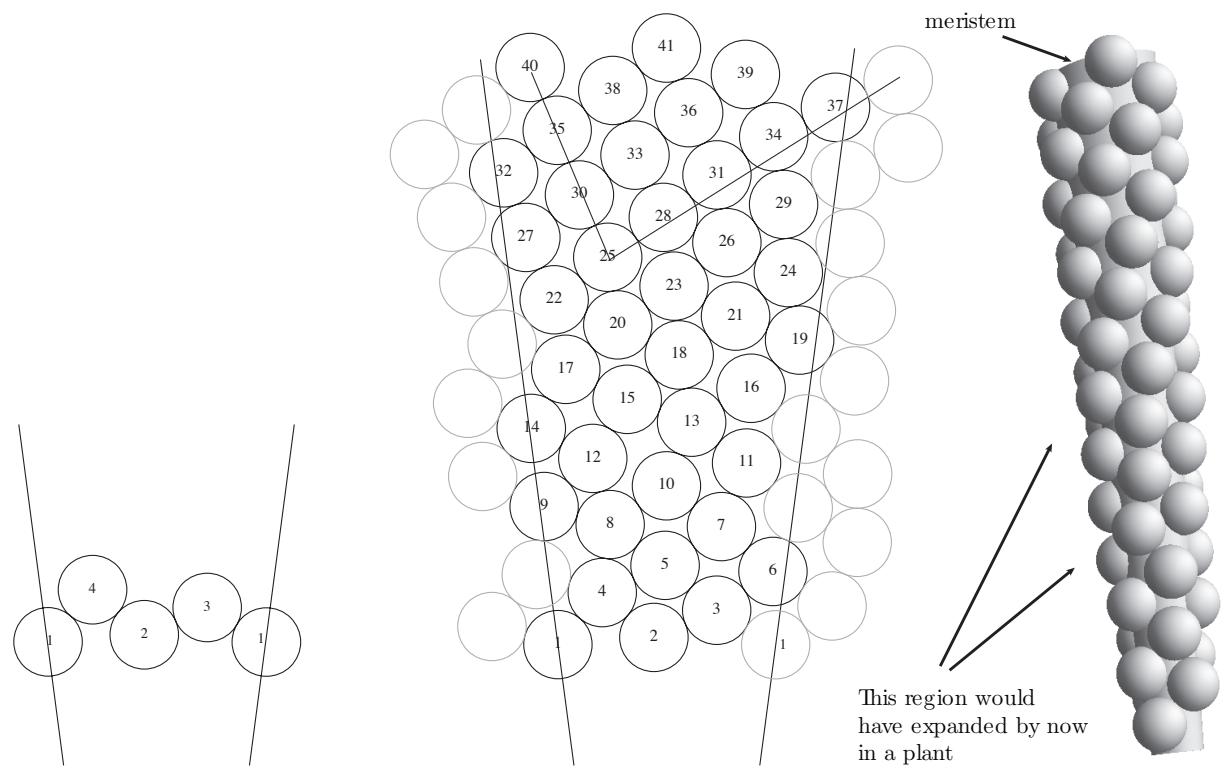

Figure 6: On the left, the dynamic geometric model in its initial stages. The initial configuration is that of circles 1 and 2. More circles are then placed, one at a time, in the lowest possible spot avoiding overlaps. Notice that at the edge of the meristem (at the top), the phyllotactic pattern is Fibonacci $(3,5)$, as it is indicated using circle 40 as reference. It evolves to Figure 9a. On the right, the configuration viewed "around the stem". In a plant the lower part, away from the meristem, would have also expanded in size, preserving the pattern, just as one sees in Figure 2.

From now on, we will deal with configurations that arise starting with exactly two different circles. The initial position of these two first circles determines an outcome. An example is shown in Figure 6, where circles numbered 1 and 2 determine the configuration that arises. After a few iterations the configuration evolves in this case into rising Fibonacci phyllotaxis. In the figure on the right we see what the configuration looks like when seen wrapped around the stem. In a plant the lower part, away from the meristem, would have also expanded considerably in size, but preserving the pattern, just as one sees in Figure 2.

Different initial positions result in different outcomes, i.e., sometimes Lucas numbers, bijugate configurations and even multijugate configurations (see Figure 7). We will see more about this in the next section.

We emphasize that the two postulates make any of these phyllotactic patterns to progressively evolve as a "fibonacci-like" sequence, with each number being the sum of the previous two. That 

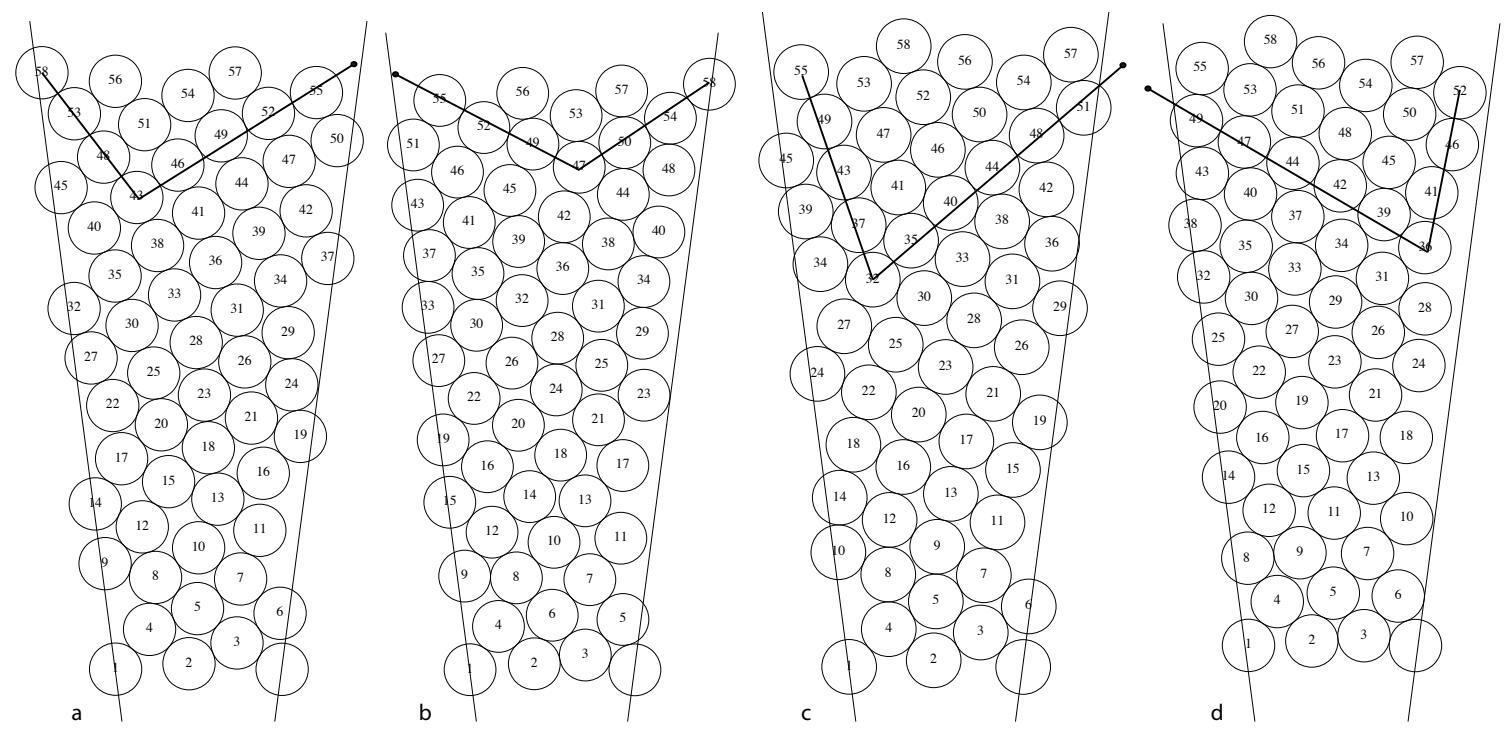

Figure 7: Different initial positions result in different configurations. Circle 1 is fixed, circle 2 is varied. (a) is the Fibonacci configuration of Figure 6. (b) Lucas, with $(3,4)$ at the top. (c) Bijugate with $(4,6)$ at the top. (d) Multijugate $(3,6)$.

is, in each case, the phyllotactic type of the pattern follows a sequence of pairs of numbers

$$
(m, n) \rightarrow(m+n, n) \rightarrow(m+n, m+2 n) \rightarrow \ldots
$$

We will see how these transitions occur in the next section.

Primordia formation with the meristem's diameter stabilized at a certain size is modeled by extending the "top" of the cone with a cylinder. When the meristem stops expanding and primordia keep appearing, the phyllotactic type of the pattern remains constant even if the structure away from the meristem expands to macroscopic level. An example is shown in Figure 8. If, on the contrary, we continue with meristem expansion, we observe that the pattern transitions further to $(8,5),(8,13),(21,13),(21,34), \ldots$ as we add more and more circles.

\section{Varying Initial Positions. Parameter Space}

We fix the angle of the wedge arbitrarily at $15^{\circ}$ (other angles give similar results). As mentioned before, different initial positions of the first two circles result in different outcome configurations. We found mainly four types of outcomes:

Fibonacci: 1, 2, 3, 5, 8, 13, 21, ..,

Lucas: $1,3,4,7,11,18,29, \ldots$

Bijugate: 2, 4, 6, 10, 16, 26, 42, ..

Multijugate (Trijugate): 3, 6, 9, 15, 24, 39, ..

The bijugate numbers are two times the Fibonacci numbers and the trijugate are three times these. We note that in very few unstable isolated boundary cases we got, for example $5,7,12, \ldots$. 


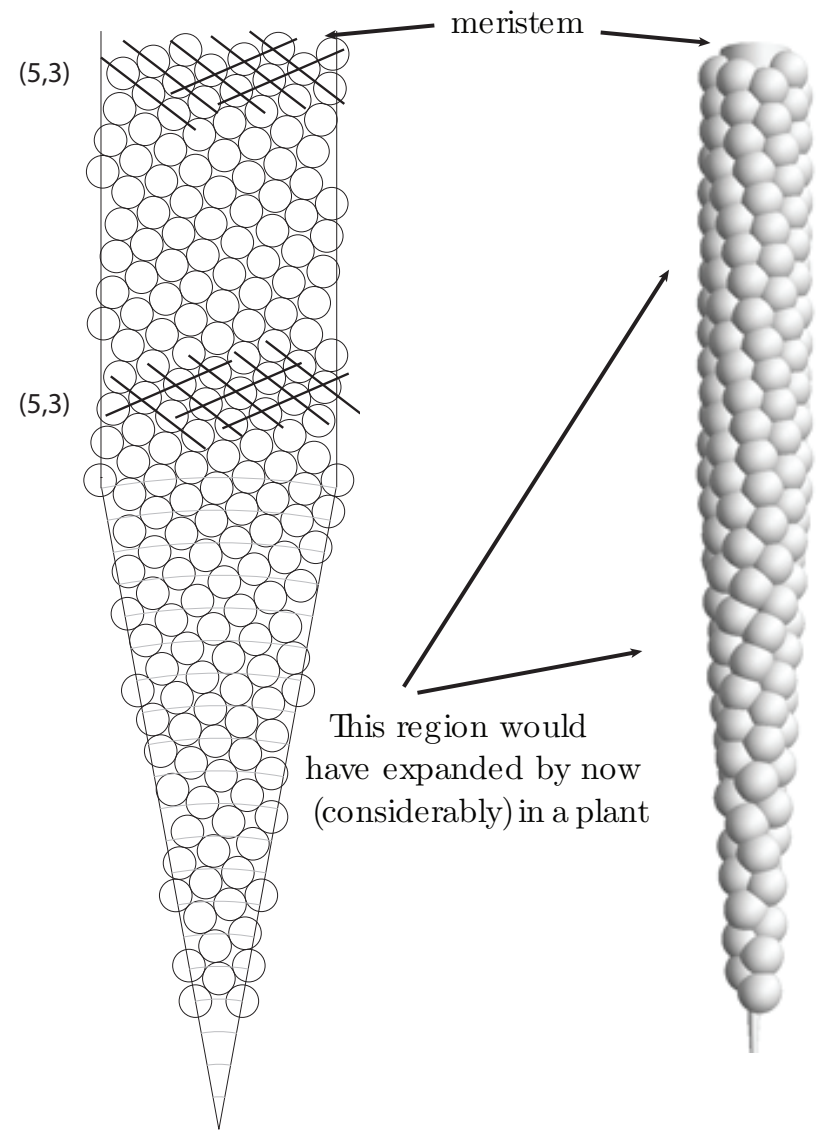

Figure 8: Experiment implementing the meristem expansion postulate and then, after a while, the stabilization of its size. While meristem expands, we have rising phyllotaxis as we can see in the lower part: $(1,1) \rightarrow(2,1) \rightarrow(2,3) \rightarrow$ $(5,3)$. When meristem stops expanding, we can see a constant phyllotactic pattern $(5,3)$ or $(5,3,8)$. On the right, we chose a different way to render primordia than in Figure 6 making the bumps a little larger but still at the exact location of the circles. Again, in a plant, the region below the meristem would have grown and expanded greatly but preserving the phyllotactic pattern.

Figures 9 and 10 show the four examples of Figure 7 with 312 circles. We chose these as arbitrary representatives of the four types we find. We took the radius of the circles as the unit of measure. In all four examples, circle 1 is in the same place, at a distance 24.4 from the vertex. Circle 2 is in different locations. Figure 11 shows the top layer of these four configurations after 1200 circles.

Notice how the patterns evolve. Take the example in Figure 9(a). A region with two clear sets of parastichies and phyllotactic pattern $(m, n)=(3,5)$ has each circle being tangent to exactly two below it (e.g., circle 56 has 53 and 51). The pattern evolves with a third set of parastichies becoming more and more distinct until we reach a brief transition region, where we have a few triple tangencies (a circle tangent to three circles below it, e.g., circle 83 has 79, 75 and 78 below it) and three sets of parastichies with numbers $m, n$ and $m+n$. A bit later, after the transition, the pattern becomes $(m+n, n)=(8,5)$. 
$(8,13)$

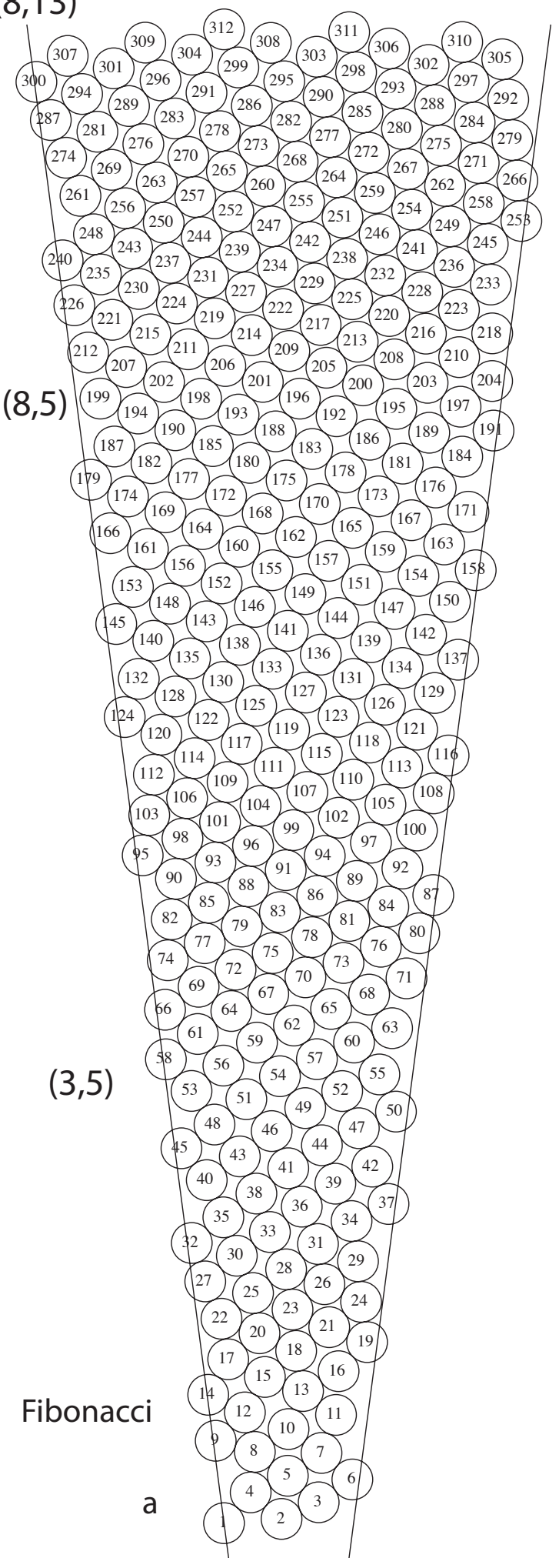

$(11,7)$

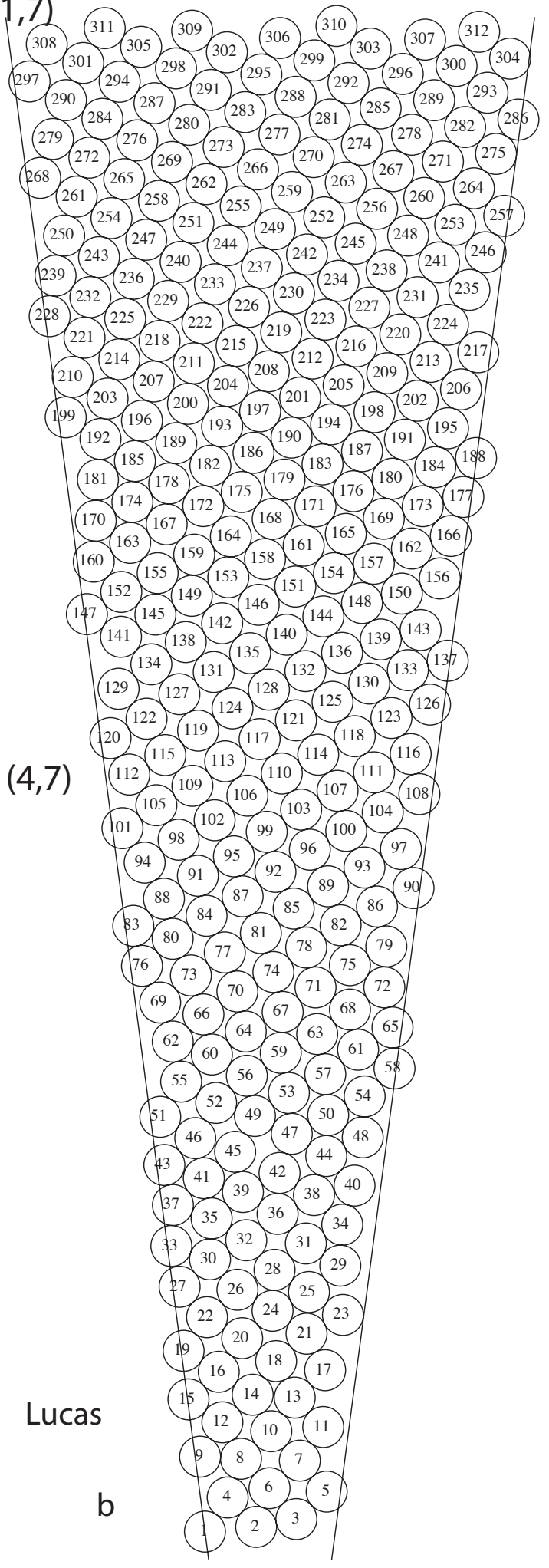

Figure 9: Here and in the next figure, the same four configurations of Figure 7 but with 312 circles. In (a) circle 56 and others have only two tangent circles below it, and circle 83, which is in a transition region, has three. 



Figure 10: Here and in the previous figure, the same four configurations of Figure 7 but with 312 circles. 

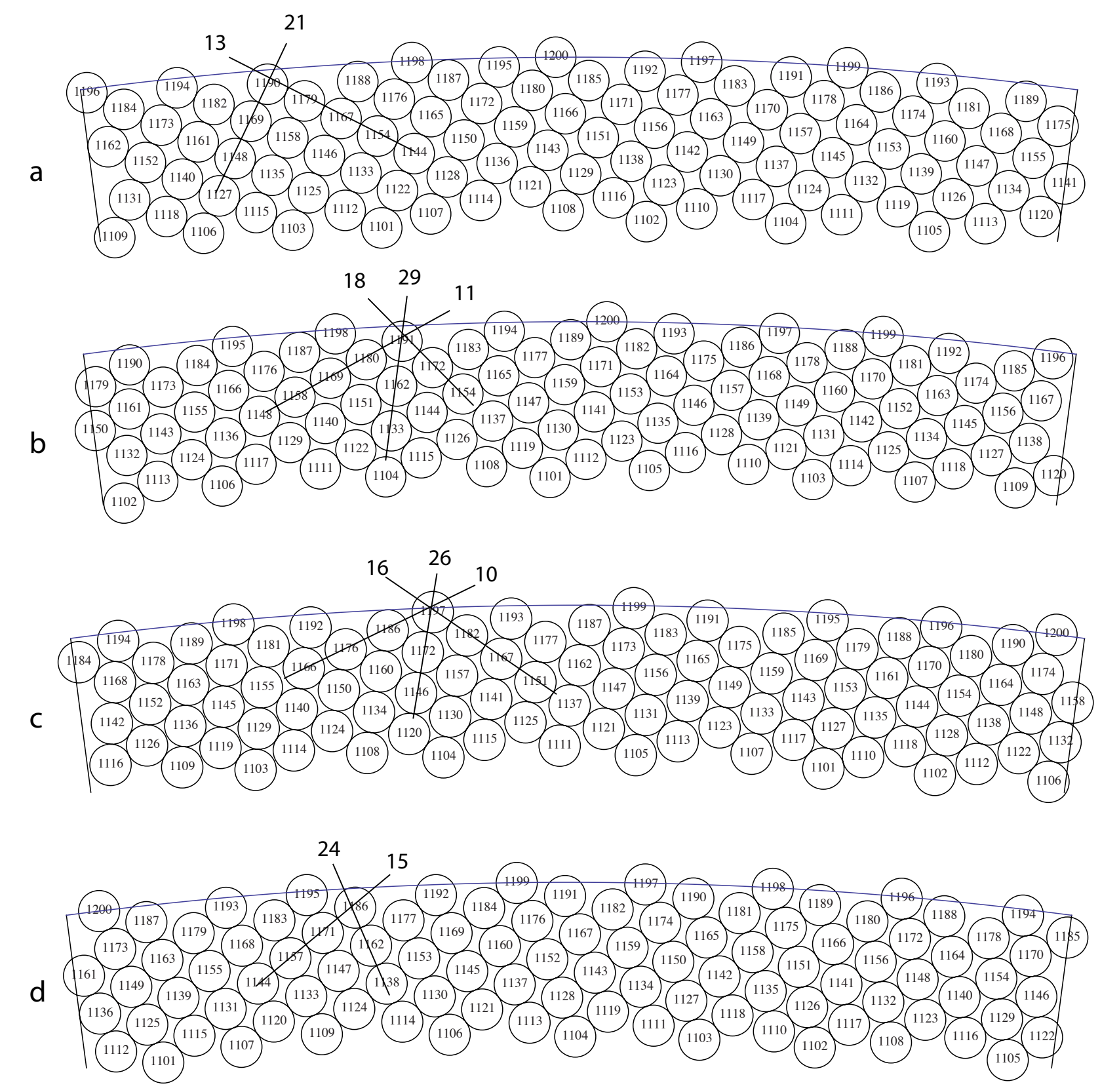

Figure 11: The configurations of Figures 7, 9 and 10 after 1200 circles. Only the top portion is shown. (a) Fibonacci $(21,13)$. (b) Lucas $(11,18)$ in transition with the third set of spirals with number 29, but circles non-tangential. (c) Bijugate in transition $(10,16,26)$. (d) Multijugate $(15,24)$. 


\subsection{Parameter Space. The Lense-Spaces}

We study now systematically the set of initial positions of two circles- the "parameter space." As we mentioned before, the position of these two initial circles 1 and 2 determines the outcome. We take the radius of the circles as the unit of measure. We ask that the centers of circles 1 and 2 be less than two diameters apart (distance $=4$ ) on either side (recall that we are on a cone) so that circle 3 (and perhaps also circle 4) can be placed tangent to both. Fixing the "height" parameter $h$ of circle 1, which is its distance to the cone's vertex, we have then a two-dimensional lense-shaped cross section of parameter space as the region of possibilities where circle 2 may be placed (see Figure 12). We refer to these as "lense spaces."
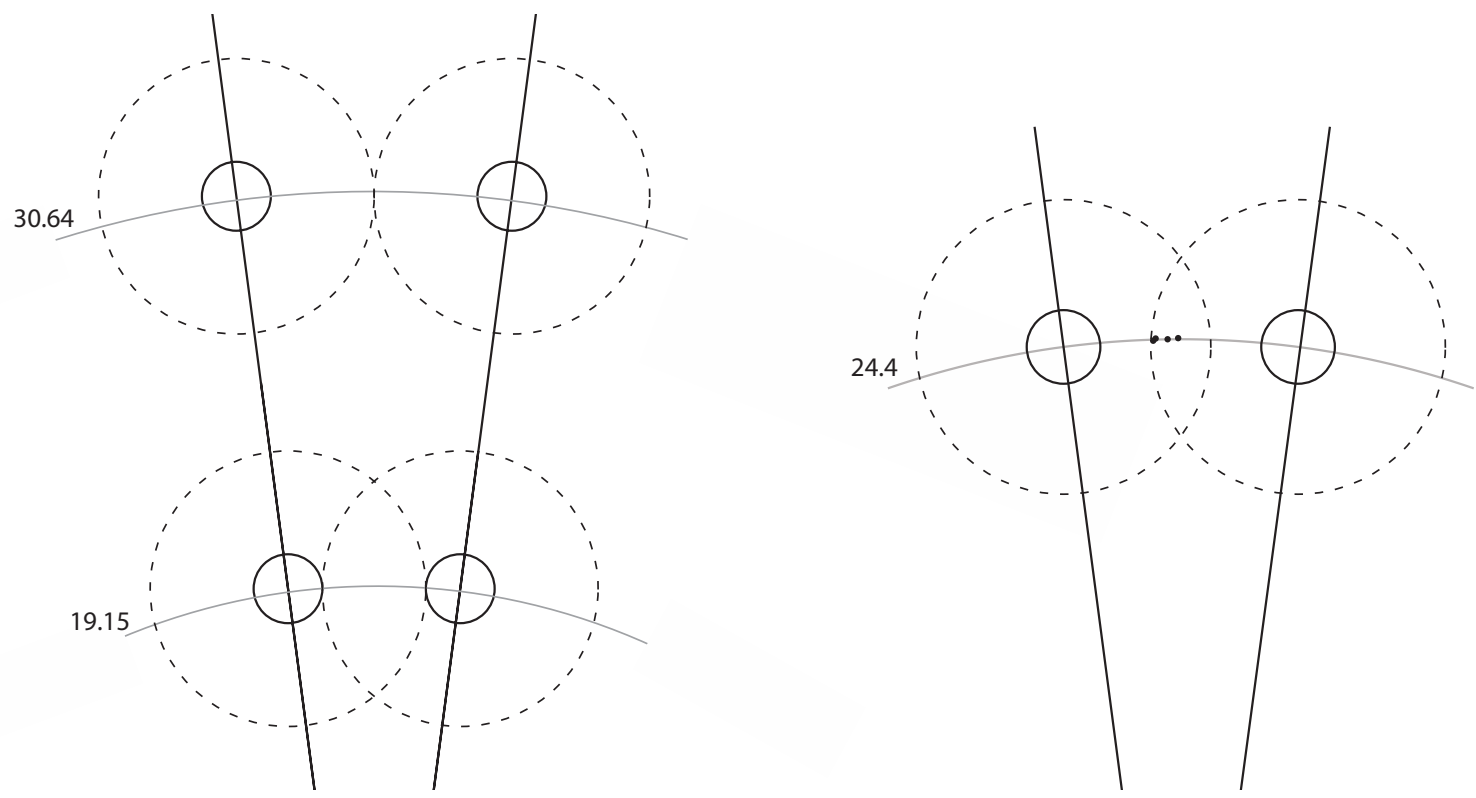

Figure 12: Parameter space. On the left, the two extremes in height $h$ for placing circle $1, h=30.64$ and $h=19.15$. The dotted regions around circle 1 denote a distance of less than 4 , where circle 2 can be placed. (Some locations circle 2 overlaps with circle 1.) Thus, the intersection of the two dotted circles form a lense-shaped region where circle 2 can be placed without leaving too wide a space in either side with circle 1 . On the right, $h=24.4$ with the four positions of circle 2 of Figures 7, 9 and 10 (see also Figure 13).

The four configurations in Figures 7, 9 and 10 have circle 1 at same location $h=24.4$. So, these four configurations are represented by four corresponding points in the lense $h=24.4$. These four points are the centers of circle 2 (one for for each case) and they are plotted in Figures 12 and 13.

In Figure 13 we scanned this lense-space (with $\Delta=.007$ in both directions) and color-coded each point according to the phyllotactic type of the outcome after 300 iterations: (f) Fibonacci (the large white spaces), (l) Lucas, (b) Bijugate, and (m) Multijugate (trijugate).

Figure 14 shows the results of scanning the lense spaces $h=19.16$ and $h=22.16$, the large white regions within the lenses correspond to Fibonacci cases. For $h=19.16$ there are only Fibonacci and bijugate configurations.

We scanned the lense spaces at intervals $\Delta h=0.2$ using $\Delta=0.007$ in both directions. Figure 15 shows four other scanned lense spaces, corresponding to $h=23.6,24.2,24.8$ and 25.4. 


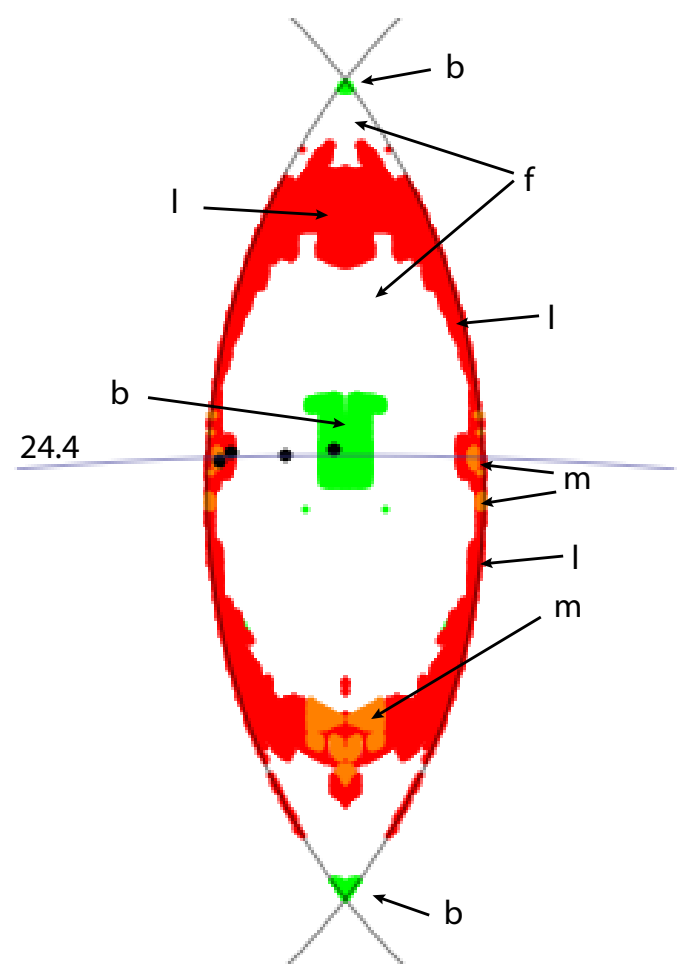

Figure 13: Lense space with circle 1 at $h=$ 24.4. Each point inside the lense represents an initial position for circle 2. (For this value of $h$, circle 2 does not ever overlap circle 1.) We scanned the lense space with $\Delta=.007$ and colored each point according to the phyllotactic type of the outcome observed. In white, and marked with (f), fibonacci configurations. (l) lucas, (b) bijugate, and (m) multijugate (in fact, trijugate). The four black points correspond to the four configurations in Figures 7, 9 and 10.
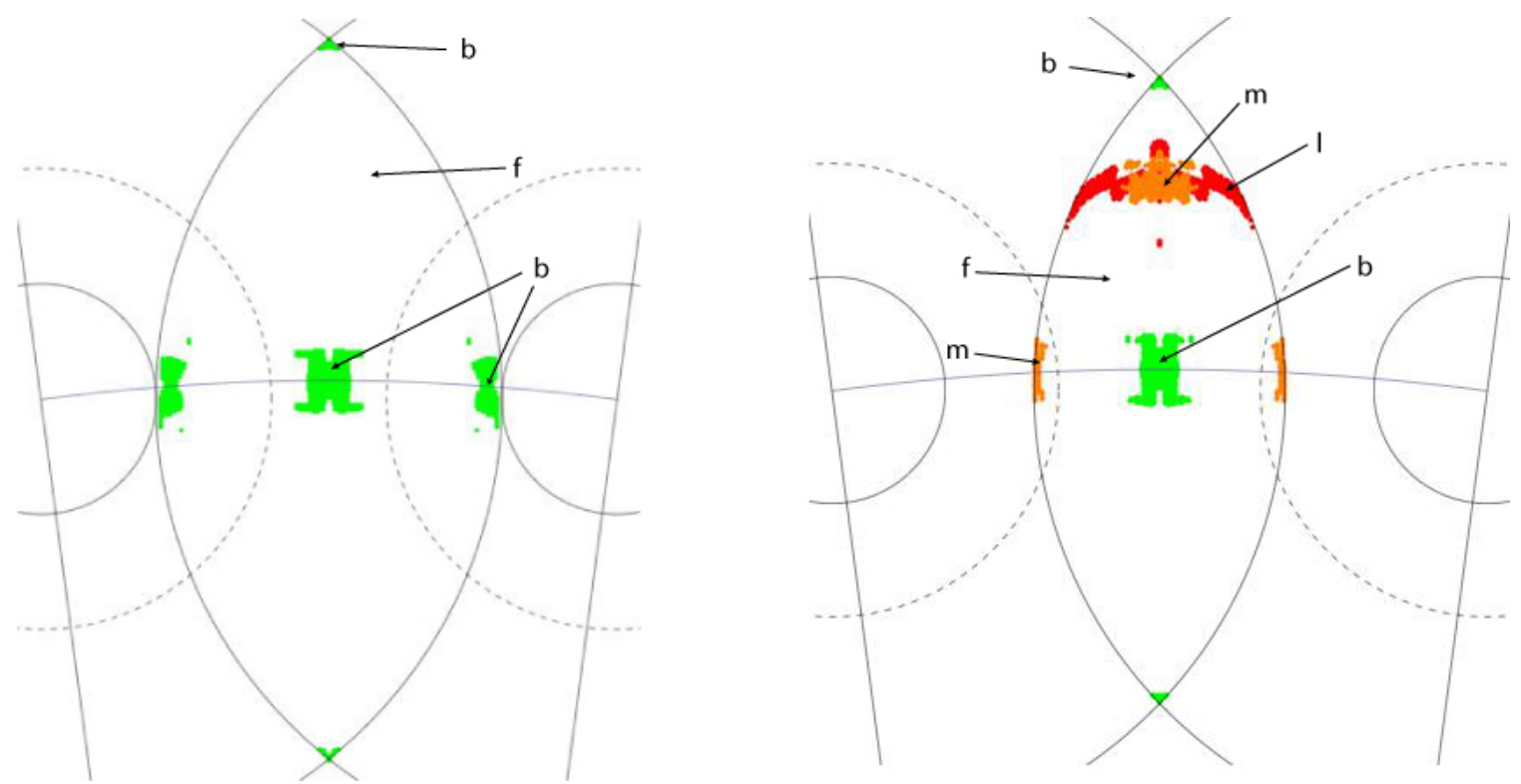

Figure 14: Two lense spaces, corresponding to $h=19.16$ on the left, and $h=22.16$ on the right . Circle 1 is placed on the edge of the wedge as we did in Figure 12. The dotted circles here denote regions where circle 2 would overlap with circle 1. 


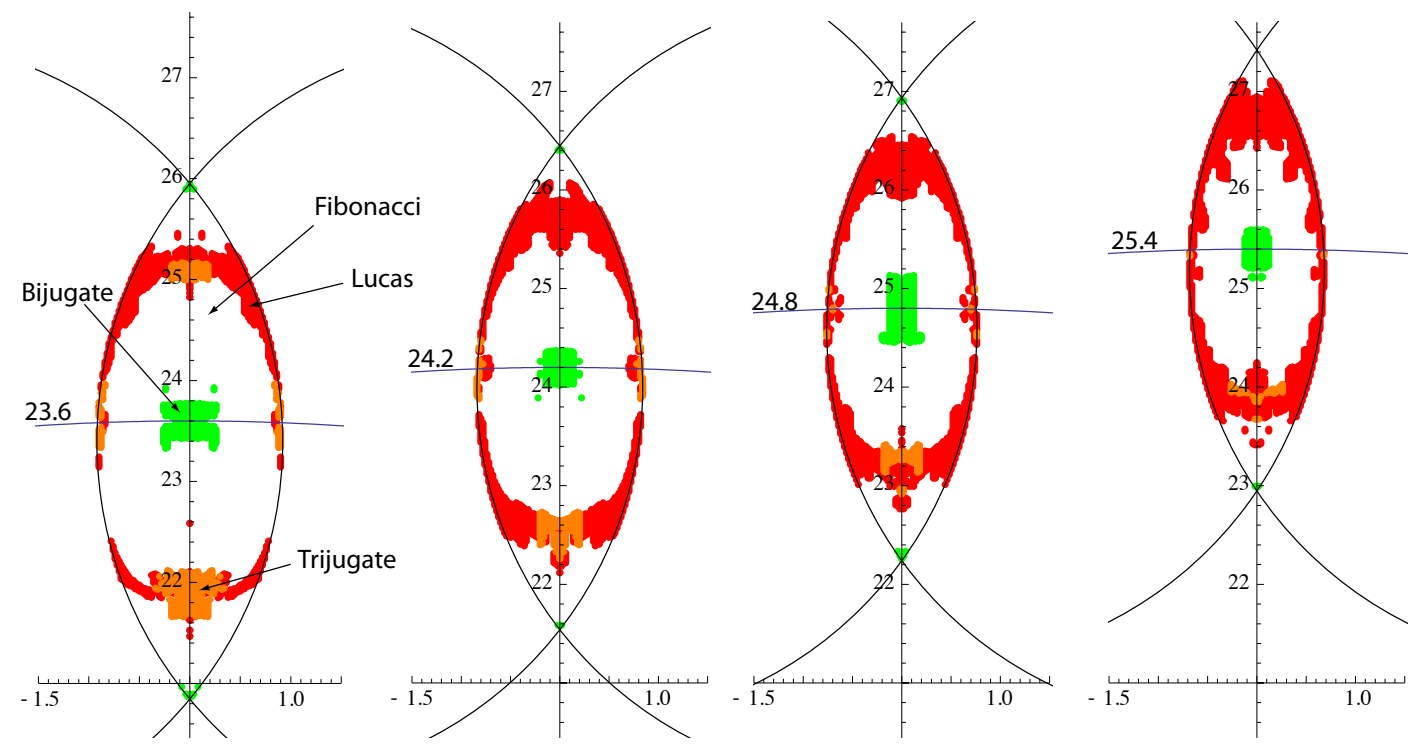

Figure 15: Four lense spaces, corresponding to $h=23.6,24.2,24.8$ and 25.4. For these values of $h$, circle 2 does not overlap with circle 1 when placed within the lense.

\section{References}

[1] P. Atela, C. Gole, S. Hotton. A dynamical system for plant pattern formation: a rigorous analysis. J. Nonlinear Sci., 12 (2002), No. 9, 641-676.

[2] S. Douady, Y. Couder. Phyllotaxis as a physical self organized growth process. Phys. Rev. Lett., (1992), No. 68, 2098-2101.

[3] S. Douady, Y. Couder. Phyllotaxis as a Self Organizing Iterative Process, Parts I, II and III. J. Theor. Biol., (1996), No. 178, 255-312.

[4] W. Hofmeister. Allgemeine Morphologie der Gewachse, in Handbuch der Physiologishcen Botanik. Engelmann, Leipzig, (1868), No. 1, 405-664.

[5] S. Hotton, V. Johnson, J. Wilbarger, K. Zwieniecki, P. Atela, C. Gole, J. Dumais. The possible and the actual in phyllotaxis: Bridging the gap between empirical observations and iterative models. Journal of Plant Growth Regulation, (2006), No. 25, 313-323.

[6] R. V. Jean. Phyllotaxis, a Systemic Study in Plant Morphogenesis. Cambridge University Press, 1994.

[7] R.V. Jean, D. Barab, editors. Symmetry in Plants. World Scientific, Singapore, 1994.

[8] L.S. Levitov. Energetic Approach to Phyllotaxis. Europhys. Lett., 14 (1991), No. 6, 533539.

[9] M. Snow, R. Snow. Minimum Areas and Leaf Determination. Proc. Roy. Soc., (1952), No. B139, 545-566.

[10] G. van Iterson. Mathematische und Microscopisch-Anatamische Studien über Blattstellungen, nebst Betrshungen über den Schalenbau der miliolinen. G.-Fischer-Verlag, Jena, 1907. 